\title{
Measurement of Arterio-Venous Ratio for Detection of Hypertensive Retinopathy through Digital Color Fundus Images
}

\author{
${ }^{1}$ Anju Rani and ${ }^{2}$ Dr. Deepti Mittal \\ ${ }^{1}$ Department of Electrical and Instrumentation Engineering, Thapar University, Patiala, India \\ 1anju.yaduvanshi@yahoo.com; ²deepti.mittal@thapar.edu
}

\begin{abstract}
Hypertensive retinopathy is a retinal vascular damage caused by high blood pressure which results in loss of vision. In the present work, effort has been devoted to enhance and segment the retinal vasculature which is required to calculate its anatomical characteristics such as width, length for the quantitative measurement of arterio-venous ratio (AVR). Enhancement of the retinal fundus images is done using tophat transform and segmentation of the vessels using iterative thresholding. The performance of the proposed method is tested on 50 digital fundus images of publicly available MESSIDOR dataset. The hypertensive retinopathy can be measured quantitatively by detecting the decrement in the ratio of width of retinal artery-vein to that of the normal images. The arterio-venous ratio obtained by applying the proposed methodology was found to be $0.62-0.735$ in normal cases and 0.203-0.495 in case of patients suffering from hypertensive retinopathy on MESSIDOR dataset which was not performed earlier. This measurement of arterio-venous ratio will be further helpful to identify the stages of hypertensive retinopathy.
\end{abstract}

Keywords: Hypertensive retinopathy; fundus image; arterio-venous ratio; arterio-venous nicking

\section{Introduction}

The abnormality of retina caused by high blood pressure is called hypertensive retinopathy. When the blood pressure is too high, the retina's blood vessel walls may thicken. This may result in narrowing of the vessels, which restricts blood to reach up to retina. Hypertensive retinopathy (HR) causes various changes in the retinal blood vessels such as leakage from blood vessels, and swelling in other parts of the retina. Figure1 (a) shows major parts of retina such as optic disc, macula, artery, vein while Figure1 (b) represents various effects of HR such as vascular wall changes, flame-shaped hemorrhages, cotton-wool spots, yellow hard exudates, and papilloedema [1,2]. Prolonged high blood pressure can cause damage to the retina's blood vessels and limit the retina's function, and can also put pressure on the optic nerve that may lead to complete vision loss. Worldwide, hypertensive retinopathy is now a leading cause of disability and mortality. Its prevalence is expected to reach $30 \%$ worldwide by 2025 . Wong et al. [2] surveyed that hypertensive patients were $50-70 \%$ more likely to have retinal hemorrhages and micro aneurysms, 30 $40 \%$ more likely to have focal arteriolar narrowing and $70-80 \%$ more likely to have arterio-venous (AV) nicking than normal individuals. Patients suffering with diabetes as well as hypertension greatly increases risk of vision loss. The automated detection of vascular changes of blood vessels is a challenging task [3]. In the present work, effort has been devoted to enhance and segment the retinal vasculature which is

DOI: 10.14738 / jbemi.25.1577

Publication Date: 27th October 2015

URL: http:/ / dx.doi.org/ 10.14738/ jbemi. 25.1577 
required to calculate its anatomical characteristics such as width, length for the quantitative measurement of arterio-venous ratio (AVR).

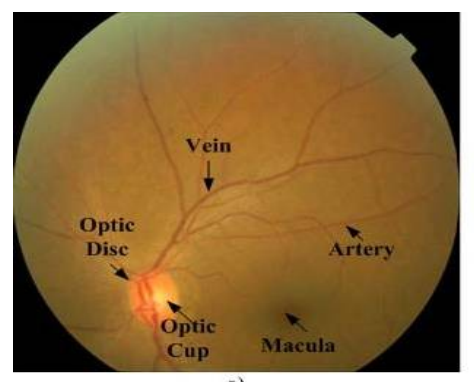

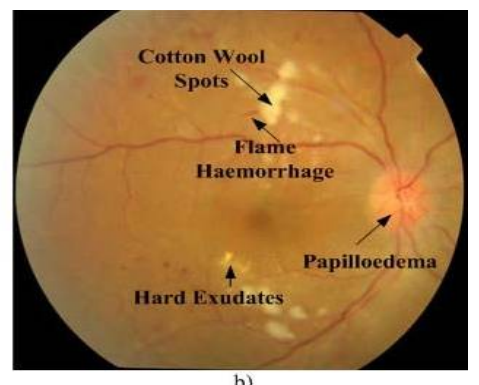

b)

Figure 1. Hypertensive retinopathy on digital fundus image: a) normal fundus image, b) fundus image showing effects of hypertensive retinopathy.

\section{Related Work}

Narasimhan et al. [4] presented a method for blood vessel detection using median filter and top hat transform on 76 images of VICAVR database and 25 clinically acquired retinal fundus images. Inspite of using large dataset, grading has not been done in their work. M anikis et al. [5] work was based on hessian based vessel segmentation technique along with thresholding. It was tested on two different standard databases DRIVE, STARE. The limitation of their work was that optic disc detection technique has not been discussed properly. Agurto et al. [6] developed automated vessel segmentation method based on a multiscale linear structure enhancement and the second order local entropy thresholding. Calculation of AVR is done on the six widest (major) vessels for each category on 74 clinically acquired images. The limitation of their work was that the AVR calculation has been done only on region (0.5-1) optic disc diameter (DD) which may give inappropriate results according to them. Khitran et al. [7] work was based on classification of vessels as arteries and veins using new feature vector. They tested their method on 58 fundus images of VICAVR and 40 images of DRIVE database. The limitation of their work was that new computation method used for AVR calculation is not discussed properly. Ortiz et al. [8] proposed a method to calculate the AVR by using different techniques including Gabor wavelet, gradients and morphological operations. A set of 30 images was taken to test this algorithm. Their method gave undefined classifications for low illuminance images. Ruggeri et al. [9] presented an algorithm to enhance the image to highlight the vessel network, which is then traced by a vessel tracking algorithm. The method was tested on 50 clinically acquired images. It appeared that some vessels were missed by the tracing procedure and therefore gave faulty AVR and thus unsatisfactory performances.

On the basis of literature review, for the best result noise removal can be done by using gaussian kernel and enhancement can be carried out using top-hat transform. This insight is helpful to design a methodology to measure AVR ratio on the images of M ESSIDOR dataset which has not been done earlier.

This paper is structured as follows: Section 3 outlines the materials used and describes the proposed method. Section 4 provides the experimental results of the proposed method and discussion on these results. Section 5 summarizes the key findings of the method and scope for future research. 
Anju Rani and Deepti Mittal; Measurement of Arterio-Venous Ratio for Detection of Hypertensive Retinopathy through Digital Color Fundus Images. Journal of Biomedical Engineering and Medical Imaging, Volume 2, No 5, October (2015) , pp 35-45

Table 1. Performance Comparison of Hypertensive Retinopathy Detection.

\begin{tabular}{|c|c|c|c|c|}
\hline \multirow{2}{*}{$\begin{array}{l}\text { Author } \\
\text { (Year) }\end{array}$} & \multirow{2}{*}{$\begin{array}{l}\text { Image processing } \\
\text { Methods }\end{array}$} & \multirow{2}{*}{$\begin{array}{c}\text { Database } \\
\text { (Number of images) }\end{array}$} & \multicolumn{2}{|c|}{ AVR } \\
\hline & & & Normal & Abnormal \\
\hline $\begin{array}{l}\text { Narasimhan et al. } \\
\text { (2012) [4] }\end{array}$ & $\begin{array}{l}\text { M edian filter, } \\
\text { Top hat transform }\end{array}$ & $\begin{array}{c}\text { VICAVR(76) } \\
\text { Clinically acquired(25) }\end{array}$ & $0.6-0.7$ & $0.24-0.49$ \\
\hline $\begin{array}{l}\text { Manikis et al. } \\
\text { (2011) [5] }\end{array}$ & $\begin{array}{c}\text { CLAHE, } \\
\text { Hessian based vessel segmentation }\end{array}$ & $\begin{array}{l}\text { DRIVE(40) } \\
\text { STARE(20) }\end{array}$ & - & $0.1-0.5$ \\
\hline $\begin{array}{l}\text { Agurto et al. } \\
\text { (2014) [6] }\end{array}$ & $\begin{array}{c}\text { Multi-scale linear structure, } \\
\text { enhancement }\end{array}$ & Clinically acquired(74) & 0.67 & 0.58 \\
\hline $\begin{array}{l}\text { Khitran et al. } \\
\text { (2014) [7] }\end{array}$ & $\begin{array}{c}\text { Gabor wavelet, } \\
\text { multilayered thresholding }\end{array}$ & $\begin{array}{l}\text { VICAVR(58) } \\
\text { DRIVE(40) }\end{array}$ & - & $0.1-0.5$ \\
\hline $\begin{array}{l}\text { Ortiz et al. } \\
(2010)[8]\end{array}$ & $\begin{array}{l}\text { Gabor wavelet, } \\
\text { Hessian matrix }\end{array}$ & Clinically acquired(50) & $>0.6$ & $<0.6$ \\
\hline $\begin{array}{l}\text { Ruggeri et al. } \\
(2007)[9]\end{array}$ & Vessel tracking method & Clinically acquired(50) & - & $\varangle 0.57$ \\
\hline
\end{tabular}

\section{Materials and Methods}

The retinal fundus images for the present study have been taken from open-source benchmark M ESSIDOR database which is available online. MESSIDOR database were acquired by three ophthalmologic departments using a color video 3CCD camera on a Topcon TRC NW6 non-mydriatic retinograph with a 45 degree field of view. The images were captured using 8 bits per color plane at $1440 \times 960,2240 \times 1488$ or $2304 \times 1536$ pixels. The database contains a medical diagnosis for each image, but no manual annotations on the images, such as lesions contours or position.

The flowchart of the proposed method is presented in figure 2. Hypertensive retinopathy produces modifications in the retinal vessels; hence the present work is focused mainly on vessel enhancement and segmentation to characterize any morphological change in retina. Therefore, the main stages involved in this method are: (i) pre-processing step which mainly includes vessel enhancement process, (ii) postprocessing step having vessel segmentation, binarization and optic disc detection as its main step, (iii) retinal tree labeling to perform characteristic measurements on composite image (iv) ROI detection to perform AVR calculation.

\subsection{Pre-processing}

The retinal fundus image consists of three channels; green, red, and blue. Amongst them, green channel is selected which has a better contrast and information than the other two channels.

Retinal blood vessels exhibit number of properties such as:

i. The blood vessels have small curvature.

ii. Vessels have lower reflectance than the background, so they appear darker.

iii. Vessel size may decrease when moving away from the optic disc, the width of a retina vessel may lie within the range of 2-10 pixels.

iv. The intensity profile may vary by a small amount from vessel to vessel.

v. The intensity profile of blood vessel has a Gaussian shape [10].

So the blood vessels are enhanced and segmented for further processing using following major steps:

\subsubsection{Vessel central light reflex removal}

Some blood vessels have a light streak (known as a light reflex) which runs down the central length of the blood vessel [4].The green plane of the fundus image is filtered to remove this brighter strip. So, 
morphological opening is applied to the green channel using a three-pixel diameter disc as structuring element. The structuring element is selected such that it does not merge with the vessels that lie close to one another.

\subsubsection{Background homogenization}

The second step of preprocessing is background homogenization. A background intensity variation due to non-uniform illumination is associated with retinal fundus images. A 3x3 mean filter is applied to smooth occasional salt-and-pepper noise [11]. The image is smoothened further with a gaussian kernel with standard deviation of 0.5 . A homogenized image in then obtained with reduced intensity variations in accordance to the green channel image.

\subsubsection{Vessel enhancement}

Vessel enhancement is the final pre-processing step to generate a new vessel enhanced image. It is performed initially by estimating the complementary image of the homogenized image $[12,13]$. It is further followed by morphological top-hat transformation of the complementary image. A disc structuring element of eight pixels radius was used in this step. The bright lesions i.e., optic disc, possible presence of exudates or reflection artifacts are removed while the dark features i.e., blood vessels, fovea, possible presence of micro aneurysms or hemorrhages remain in the image. The contrast between the vessels and other tissues are further improved after applying top-hat transform.

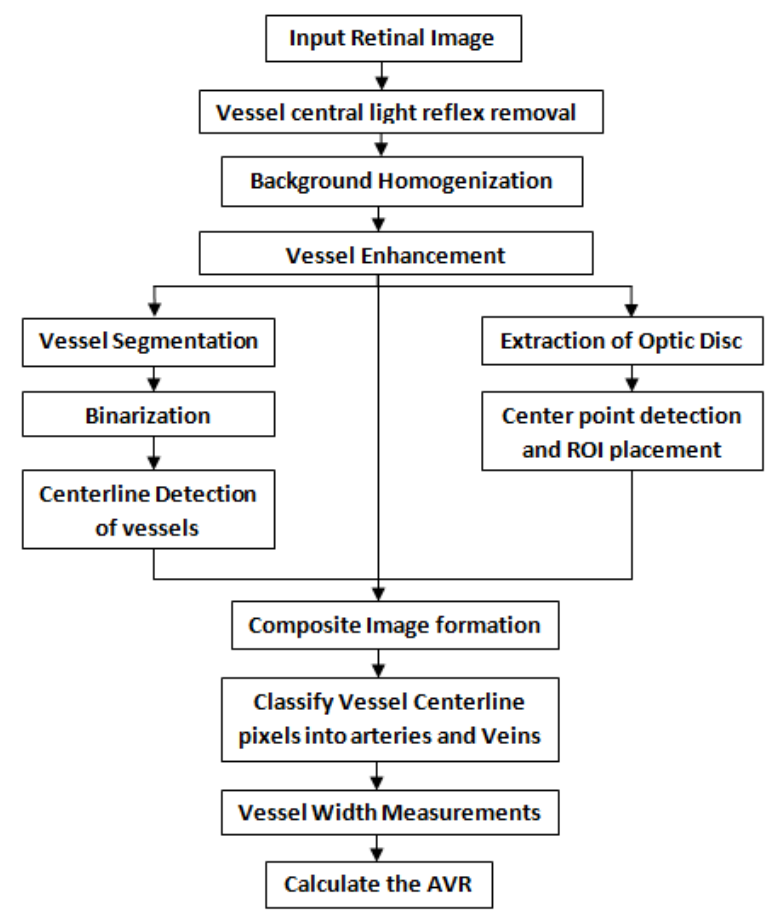

Figure 2. Flowchart of the proposed method 
Anju Rani and Deepti Mittal; Measurement of Arterio-Venous Ratio for Detection of Hypertensive Retinopathy through Digital Color Fundus Images. Journal of Biomedical Engineering and Medical Imaging, Volume 2, No 5, October (2015) , pp 35-45

\subsection{Post-processing}

\subsubsection{Vessel Segmentation}

Classification of arteries and veins is an important step in HR detection. An iterative thresholding method for segmenting the blood vessel structure is applied for the binarization of the enhanced image $[14,15,16]$. Intensity variation and color information is used to classify the vessels as arteries and veins $[17,18]$.

\subsubsection{Optic disc detection and ROI Determination}

Accurate detection of optic disc is necessary for determining the region of interest (ROI) so as to calculate the artery-vein ratio [6]. The center point of the optic disc is estimated by the automated method. The estimation is based on measurements obtained in the image and from the vessel segmentation. The target location is obtained by taking estimates in many locations in the image, ignoring those locations that are estimated to be far from the optic disc center [19]. The automated method detects the center of the optic disc and a circle with DD, is placed at this location. It was assumed that this circle corresponds to the optic disc outline in the image.

After the coordinates of the optic disc are located, two regions of interest are selected. Region A is between 0.5 and $1 \mathrm{DD}$ from the optic disc center and region $B$ is between $1 \mathrm{DD}$ and $1.5 \mathrm{DD}$ from the optic disc center. The two regions selected from optic disc center are shown in Figure 3(a). All analyses and evaluations performed in this work are based on measurements within region B. Region B is generally used for vessel measurements.

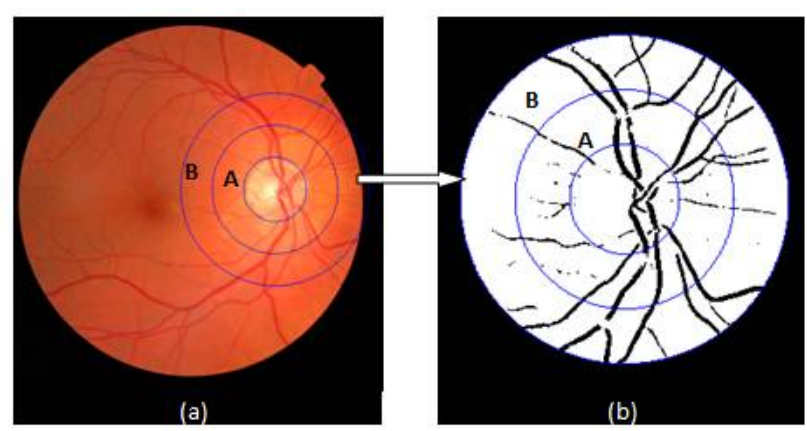

Figure 3. (a) ROI outlines marked on original image, (b) ROI in the binary image where region A is between (0.5 -1) DD and region B is between (1-1.5) DD.

\subsubsection{Computation of AVR and Centerline detection}

For computation of AVR, all the vessel width measurement has to be done initially. To calculate the vessel width from binary vessel mask, the center line for each vessel is obtained by morphological thinning operation. The thinning operation is performed till the vessel width equal to single pixel is obtained. This will provide center line for each vessel segment [8]. The vessel center line is combined with the filtered image and width is calculated for major six arteries and veins by measuring the perpendicular line that intersects the vessel edges [20]. Figure 4 represents the composite image obtained after the above process. The final width for each vessel segment is calculated as the median of all the widths of arteries and veins [6].

The AVR is computed by measuring Central Retinal Arterial Equivalent (CRAE) and Central Retina Venous Equivalent (CRVE). These two measurements of arterioles and venules are determined by Parr formulas 
[21,22] and Hubbard [23] respectively. According to [23], the mean widths of arteries and veins segments within ROI are collected in two separate lists, namely "Arteriole" and "Venule". CRAE is computed as;
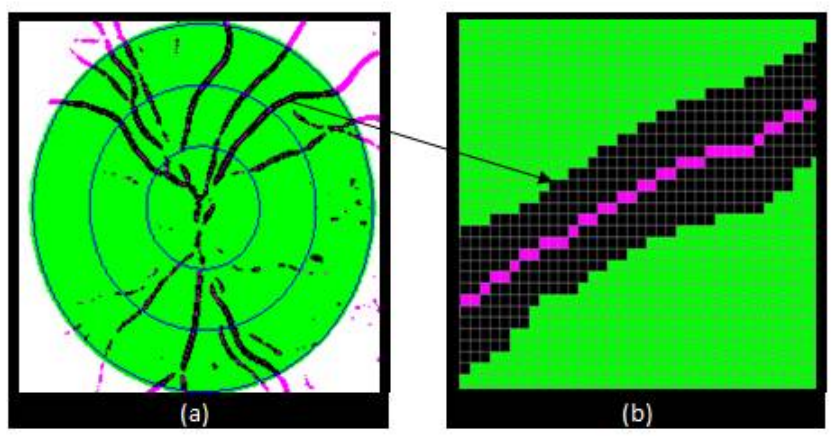

Figure 4. (a) Composite image of binarized and determined ROI image, (b) Image showing vessel width with centerline pixel.

$$
C R A E=\sqrt{\left(0.87 . X^{2}+1.01 . Y^{2}-0.22 . X . Y-10.73\right)}
$$

Where $\mathrm{Y}$ is the median value of "Arteriole" and $\mathrm{X}$ is the value in the same list exactly before the median $\mathrm{Y}$.

$$
C R V E=\sqrt{\left(0.72 . X^{2}+0.91 . Y^{2}+450.02\right)}
$$

Where $Y$ is the median of "Venule" and $X$ is the value in the list exactly before $Y$. AVR is computed by dividing equation 1 and 2 ;

$$
A V R=\frac{C R A E}{C R V E}
$$

\subsubsection{Grading of Hypertensive Retinopathy}

Keith and Wegner (1939) [24] have classified hypertensive retinopathy into following four grades. The change in vessel diameters is used to calculate AVR and it is significant in determining the presence of hypertensive retinopathy and grading its severity $[25,26]$. Table 2 shows various stages of HR along with AVR;

Table2. Grading of HR

\begin{tabular}{|c|c|c|}
\hline Degree of HR & AVR & Symptoms \\
\hline Normal Retina & $0.667-0.75$ & None \\
\hline Grade 1 & 0.5 & Mild compression of venules \\
\hline Grade 2 & 0.33 & Right angled crossing of vessels \\
\hline Grade 3 & 0.25 & All above symptoms along with papilledema \\
\hline Grade 4 & $<0.2$ & \\
\hline
\end{tabular}


Anju Rani and Deepti Mittal; Measurement of Arterio-Venous Ratio for Detection of Hypertensive Retinopathy through Digital Color Fundus Images. Journal of Biomedical Engineering and Medical Imaging, Volume 2, No 5, October (2015) , pp 35-45

\section{Experimental Results and Discussion}

The quantitative assessment of the proposed algorithm is done for hypertensive retinopathy detection. The algorithm was tested on 50 retinal images of M ESSIDOR dataset. Images in figure 5 (a), (b) and (c) are randomly chosen retinal fundus images from MESSIDOR database that are used to demonstrate the results of proposed method.

The results of pre-processing are shown in figure 5 (d)-(i). The green channel images of the selected sample images are shown in figure 5 (d), (e) and (f) respectively. The output of homogenized image is complemented to get output image as figure $5(\mathrm{~g})$, (h) and (i) respectively. The enhancement of the processed images is then done by using top hat transform shown in figure $5(\mathrm{j}),(\mathrm{k})$ and $(\mathrm{l})$ respectively. The contrast of the output obtained is then increased to get the enhanced image as shown in figure $5(\mathrm{~m}),(\mathrm{n})$ and (o) respectively. The enhanced sample images are then binarized to obtain figure 6 (a),(b) and (c) respectively.

The width of arteries and veins is calculated on region B (1-1.5) DD as show in figure 3 (a), (b). The AVR is calculated after computing the width of arteries and veins as shown in figure 4 (a), (b). Table 3 displays statistical performance of the proposed method on M ESSIDOR dataset. On the basis of Table 2, the retinal images can be divided into normal and abnormal images. The AVR obtained by the above mentioned methodology is $0.62-0.735$ in normal cases and $0.203-0.495$ in case of patients suffering from hypertensive retinopathy. Using the above algorithm 17 images out of 50 images was found to be normal images while 33 abnormal images detected the presence of hypertensive retinopathy.

In this research paper top-hat transform is used for the vessel enhancement and thresholding is used for segmentation of vessels. Optic disk detection is necessary for the correct estimation of ROI as well as for the computation of AVR. 


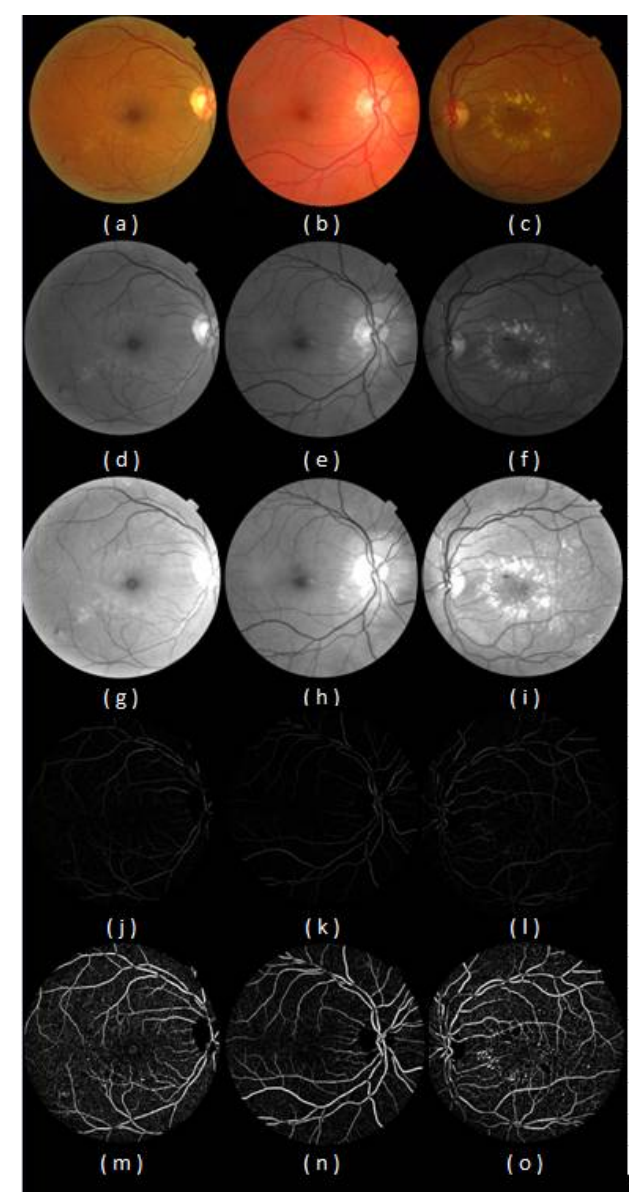

Figure5. Results of preprocessing of retinal fundus images: (a-c) original images from M ESSIDOR dataset, (df) Green channel images, (g-i) complement of homogenized output image, (j-I) output images of top-hat transform, $(m-n)$ enhanced images of top-hat transform outputs.

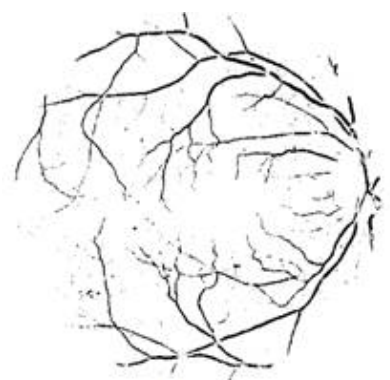

(a)

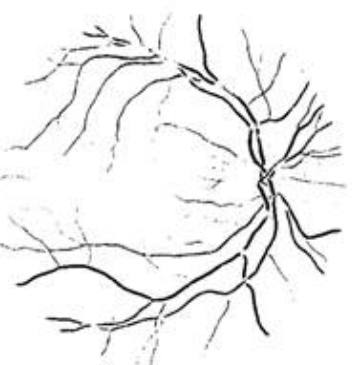

(b)

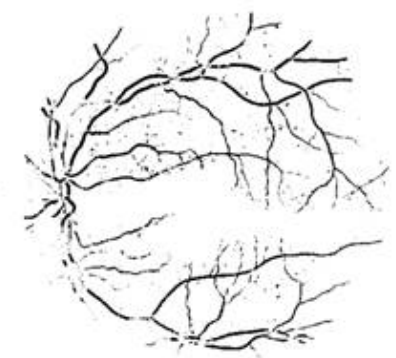

(c)

Figure 6. Binarization of enhanced image taken from sample images (a-c) 
Anju Rani and Deepti Mittal; Measurement of Arterio-Venous Ratio for Detection of Hypertensive Retinopathy through Digital Color Fundus Images. Journal of Biomedical Engineering and Medical Imaging, Volume 2, No 5, October (2015) , pp 35-45

Table3. Evaluation of the proposed method by statistical performance measures.

\begin{tabular}{|c|c|}
\hline Number of Images & AVR \\
\hline Image 1 & 0.359 \\
\hline Image 2 & 0.7266 \\
\hline Image 3 & 0.3584 \\
\hline Image 4 & 0.3841 \\
\hline Image 5 & 0.2034 \\
\hline Image 6 & 0.3548 \\
\hline Image 7 & 0.6258 \\
\hline Image 8 & 0.6188 \\
\hline Image 9 & 455646 \\
\hline Image 10 & 0.2674 \\
\hline . & . \\
\hline . & . \\
\hline . & $\cdot$ \\
\hline . & . \\
\hline . & . \\
\hline Image 44 & 0.3799 \\
\hline Image 45 & 0.3061 \\
\hline Image 46 & 0.2864 \\
\hline Image 47 & 0.3404 \\
\hline Image 48 & 0.5392 \\
\hline Image 49 & 0.6417 \\
\hline Image 50 & 0.495 \\
\hline Average & Normal -(0.62-0.735) \\
\hline & $\begin{array}{c}\text { Abnormal-(0.203- } \\
0.495)\end{array}$ \\
\hline
\end{tabular}

\section{Conclusion}

Early detection of hypertensive retinopathy signs is an important step in the risk stratification of hypertensive patients for medical as well as researcher's domain. Therefore, a new and easy method is developed for the enhancement and detection of blood vessels for the purpose of detection of hypertensive retinopathy. The AVR obtained by the proposed methodology for M ESSIDOR dataset is 0.62 0.735 in normal cases and 0.203-0.495 in case of patients suffering from hypertensive retinopathy

In future work, automated classification of blood vessels can be introduced. Evaluations on large set of images acquired from the subjects with much wider variations of AVR can be performed to access the reliability and clinical applicability of this algorithm.

\section{REFERENCES}

[1] T.Y. Wong, P. M itchell, Hypertensive retinopathy. NEJM 2004;351:2310-7.

[2] T.Y. Wong, R. Klein, B.E.K. Klein, J.M. Tielsch, L. Hubbard, and F.J. Nieto, Retinal microvascular abnormalities and their relationship with hypertension, cardiovascular disease, and mortality. Survey Ophthal 2001;46:59-80.

[3] K. Kumari and D. Mittal, Drusen Quantification for Early Identification of Age Related Macular Degeneration. Advances in Image and Video Processing, Volume 3 No 3, June (2015); pp: 28-40 
[4] K. Narasimhan , V.C. Neha, K.Vijayarekha, Hypertensive Retinopathy Diagnosis from Fundus Images by Estimation of AVR. Procedia Engineering 12/2012; 38:980-993. DOI: $10.1016 /$ j.proeng.2012.06.124

[5] G.C. M anikis, V. Sakkalis, X. Zabulis, P. Karamaounas, A.Triantafyllou, S. Douma, C. Zamboulis, K. Marias, An Image Analysis Framework for the Early Assessment of Hypertensive Retinopathy Signs. Proceedings of the 3rd International Conference on E-Health and Bioengineering - EHB 2011,24th-26thNovember, 2011, laşi, Romania

[6] C. Agurto, V. Joshi, S. Nemeth, P. Soliz, S. Barriga, Detection of hypertensive retinopathy using vessel measurements and textural features. Conf Proc IEEE Eng M ed Biol Soc 2014 ; 978-1-42447929-0/14

[7] S. Khitran, M.U. Akram, A. Usman, U Yasin, Automated System for the Detection of Hypertensive Retinopathy. Image Processing Theory, Tools and Applications (IPTA), 10.1109/IPTA.2014.7001984

[8] D. Ortíz, M. Cubides, A. Suarez, M. Zequera, J. Quiroga, Dr.J.A. Gómez, Dr. N. Arroyo, Support System for the Preventive Diagnosis of Hypertensive Retinopathy. 978-1-4244-4124-2/ 10 @2010 IEEE

[9] A.Ruggeri, E. Grisan, M.D. Luca, An automatic system for the estimation of generalized arteriolar narrowing in retinal images. Proceedings of the 29th Annual International Conference of the IEEE, France. 1-4244-0788-5/07

[10] V.P. Patil, P.R. Wankhede, Pre-Processing Steps for Segmentation of Retinal Blood Vessels. International J ournal of Computer Applications (0975 - 8887) Volume 94 - No 12, M ay 2014

[11] K.Kumari and D.Mittal, Automated Drusen Detection Technique for Age-Related Macular Degeneration. Journal of Biomedical Engineering and Medical Imaging, Volume 2, No 1, Feb (2015) , pp 18-26

[12] D. M ittal , V. Kumar , S.C. Saxena , N. Khandelwal , N. Kalra, Enhancement of the ultrasound images by modified anisotropic diffusion method. Med Biol Eng Comput (2010) 48:1281-1291

[13] K. Sharma and D. M ittal, Contrast Enhancement Technique for CT Images. Journal of Biomedical Engineering and Medical Imaging, Volume 2, No 1, Feb (2015) , pp 44-50

[14] J. Kaur and D. Mittal, Segmentation and Measurement of Exudates in Fundus Images of the Retina for Detection of Retinal Disease. Journal of Biomedical Engineering and M edical Imaging, Volume 2, No 1, Feb (2015) , pp 27-38

[15] S.Chugh, J. Kaur, D. M ittal, Exudates Segmentation in Retinal Fundus Images for the Detection of Diabetic Retinopathy. International Journal of Engineering Research \& Technology (IJERT) ISSN: 2278-0181 Vol. 3 Issue 10, October- 2014 
Anju Rani and Deepti Mittal; Measurement of Arterio-Venous Ratio for Detection of Hypertensive Retinopathy through Digital Color Fundus Images. Journal of Biomedical Engineering and Medical Imaging, Volume 2, No 5, October (2015) , pp 35-45

[16] Fraz, M.M., Remagnino, P., Hoppe, A., Uyyanonvara, B., Rudnicka, A.R., Owen, C.G., Barman, Blood vessel segmentation methodologies in retinal images - A survey. Computer methods and programs in biomedicine 108(1), 407-433 (2012).

[17] D. M ittal, K. Kumari, Automated detection and segmentation of drusen in retinal fundus images. doi:10.1016/j.compeleceng.2015.08.014

[18] Fraz, M.M., Remagnino, P., Hoppe, A., Uyyanonvara, B., Owen, C., Rudnicka, A., Barman, Retinal Vessel Extraction Using First-Order Derivative of Gaussian and M orphological Processing. In: Bebis, G., Boyle, R., Parvin, B., Koracin, D., Wang, S., Kyungnam, K., Benes, B., Moreland, K., Borst, C., DiVerdi, S., Yi-Jen, C., Ming, J. (eds.)

[19] M. Niemeijer, X. Xu, A.V. Dumitrescu, P. Gupta, B.V. Ginneken, Automated M easurement of the Arteriolar-to-Venular Width Ratio in Digital Color Fundus Photographs. IEEE Transactions on medical imaging, vol. 30, no. 11, November 2011

[20] Fraz, M.M., Remagnino, P., Hoppe, A., Basit, A., Rudnicka, A.R., Owen, C.G., Barman, Quantification of blood vessel calibre in retinal images of multi-ethnic school children using a model based approach. Computerized Medical Imaging and Graphics 37(1), 60-72 (2013). doi:10.1016/j.compmedimag.2013.01.004

[21] J.C. Parr, G.F.S. Spears, General caliber of the retinal arteries expressed as the equivalent width of the central retinal artery. Am J Ophthalmol 1974;77:472-7

[22] J.C. Parr, G.F.S. Spears, Mathematic relationships between the width of a retinal artery and the widths of its branches. Am J Ophthalmol 1974;77:478-83

[23] L.D Hubbard, Methods for Evaluation of Retinal M icrovascular Abnormalities Associated with Hypertension/Sclerosis in the Atherosclerosis Risk in Communities Study. Ophthalmology 1999;106:2269-2280

[24] N.M . Keith, H.P. Wagener, N.W. Barker (1939), Some different types of essential hypertension: their course and prognosis. Am J Med Sci, 197, 332-43

[25] Fraz, M.M., Welikala, R.A., Rudnicka, A.R., Owen, C.G., Strachan, D.P., Barman, QUARTZ: Quantitative Analysis of Retinal Vessel Topology and size - An automated system for quantification of retinal vessels morphology. ExpertSystemswithApplications;42 (20),72217234(2015). doi: http://dx.doi.org/10.1016/j.eswa.2015.05.022

[26] Fraz, M.M., Rudnicka, A.R., Owen, C.G., Strachan, D.P., Barman, Automated Arteriole and Venule Recognition in Retinal Images using Ensemble Classification. 9th International Conference on Computer Vision Theory and Applications (VISAAP), Lisbon, Portugal, 5-8 J anuary 20142014 